\title{
Tasa de Éxito del Sistema de Mediación Prejudicial por Daño en Salud en Chile entre los Años 2005 y 2009
}

\author{
Success Rate of Pre-Trial Mediation System for Health Damage in Chile between 2005 and 2009
}

Paola Candia \& Iván Suazo Galdames

CANDIA, P. \& SUAZO, G. I. Tasa de éxito del Sistema de Mediación Prejudicial por daño en salud en Chile entre los años 2005 y 2009. Int. J. Odontostomat., 5(3):300-303, 2011.

RESUMEN: la mediación prejudicial por daño en salud fue establecida dentro de la ley 19.966 de 2004 que fijó el régimen de garantías explícitas de salud en Chile. Este procedimiento se constituyó como un mecanismo no adversarial de resolución de conflictos en salud. Se revisaron las bases de datos relativas a los procedimientos de mediación del consejo de defensa del estado y de la superintendencia de salud realizados entre los años 2005 y 2009 , se calculó la tasa de éxito, considerada como el porcentaje de casos con acuerdo en relación al total de casos aceptados para mediación. La tasa de éxito medio fue de $15,92 \%$, con valores superiores en los procedimientos realizados ante el consejo de defensa del estado, con $17,94 \%$, mientras que en los procedimientos intermediados por la Superintendencia de Salud fue de un 10,63\% para el mismo período, estas diferencias resultaron estadísticamente significativas $(p<0,05)$. Se discute acerca de las causas probables de la baja tasa de éxito y las posibles diferencias de la mediación por daños en el sistema público y privado de salud.

PALABRAS CLAVE: mediación, ley 19.966, ley AUGE, conflictos en salud, deontología.

\section{INTRODUCCIÓN}

En el marco de la reforma de salud en Chile se han establecido garantías de calidad, acceso y financiamiento de la atención a los ciudadanos, lo cual ha quedado estipulado en la Ley $n^{\circ} 19.966$, llamada también Ley AUGE, la cual reconoce a la salud como un derecho fundamental de los individuos que debe ser resguardado por el Estado (Méndez, 2009; Barría \& Saint George, 2007; Ley $n^{\circ} 19.966,2004$; Ley ${ }^{\circ}$ 20.015, 2005). Hasta el 2005 este resguardo era realizado por vía judicial, dentro de un sistema lento y que en muchas ocasiones no lograba satisfacer los intereses de quienes ponen en marcha estos mecanismos, por lo que el desarrollo de mecanismos no adversariales de resolución de conflicto podría colaborar en mejorar la resolución de los conflictos en salud (Gutiérrez, 2008; Méndez).

En este aspecto se ha considerado que "la resolución amigable de litigios corresponde a una concepción moderna de la justicia; una justicia que observa, que facilita la negociación y que busca preservar las relaciones sociales" (Rodríguez, 2008).
En este contexto, la mediación en salud busca proteger la relación médico-paciente, cuando estos últimos han sentido vulnerados sus derechos en el ámbito de la salud, especialmente cuando producto de una actividad o intervención médica se produce una consecuencia no deseada por el facultativo y que deriva en perjuicio que se traduce en un daño físico objetivo o simplemente en la percepción, por parte del paciente, de daño o incumplimiento de sus garantías (Ley $n^{\circ} 19.966$, art. № 43). El proceso de mediación, fue inaugurado el 4 de marzo del 2005 a través del mecanismo legal "Ley $n^{\circ} 19.966$, dcto. $n^{\circ}$ 47 ", Chile pasó a integrar la breve lista, junto a México, de los países que cuentan con un sistema prejudicial no confrontacional de resolución de quejas médicas (Urriola, 2006; Barría, 2005; Barría \& Saint George), en el cual respecto a las disposiciones generales en términos de mediación prejudicial en salud estipula en el articulo $1^{\circ}$ : "La mediación prejudicia es un procedimiento no adversarial, que tiene por objeto propender a que, mediante la comunicación directa entre las partes y con la interven 
ción de un mediador, ellas lleguen a una solución extrajudicial de la controversia" permitiendo a las partes en conflicto, llegar a acuerdo y velar por el cumplimiento de esta premisa (Decreto $\mathrm{N}^{\circ} 47$, MINSAL, 2005).

Esta normativa contempla diferencias para el caso del sistema público y privado de salud. En el caso de los conflictos derivados de atenciones en el sistema público de salud, la mediación está a cargo del Consejo de Defensa del Estado (CDE), procedimiento que ante este organismo es obligatorio, ineludible y gratuito, y que además según la citada ley, puede designar como mediador a uno de sus funcionarios, a otro funcionario en comisión de servicio o a un profesional que reúna los requisitos legales para ser mediador (CDE, 2011); mientras que en aquellos conflictos derivados de atenciones en el sistema privado de salud, el procedimiento se realiza con mediadores acreditados ante la Superintendencia de Salud, correspondiéndole a esta entidad solamente administrar el procedimiento de mediación, siendo en este caso un proceso particular donde se deben cancelar honorarios al mediador elegido por las partes (Superintendencia de Salud, 2011).

En ambos casos, la mediación busca disminuir la judicialización de los conflictos en salud, no obstante la importancia que esto tiene en términos económicos, de disminución de la congestión del sistema judicial y en la relación médico-paciente, poco se conoce de la efectividad de este mecanismo respecto de ese resultado, tanto en el sistema de salud público, como en el privado.

Es por ello que el propósito de nuestro estudio es ilustrar la efectividad de los procesos de mediación en salud realizados en Chile entre los años 2005 y 2009. El conocimiento de estos datos permitirá evaluar el funcionamiento del sistema de mediación en nuestro país y contribuye a desarrollar nuevas estrategias disminuyan la judicialización de los conflictos en salud.

\section{MATERIAL Y MÉTODO}

Se diseñó un estudio descriptivo de corte transversal, a partir de la información obtenida de las bases de datos del Consejo de Defensa del Estado y la Superintendencia de Salud, obtenidas en virtud de la ley de transparencia.
Se consideraron los casos de mediación llevados entre los años 2005 y 2009. Se calculó la tasa de éxito del sistema en base a la siguiente definición operacional:

Tasa de éxito: Número de casos con acuerdo a través de mediación

Número de casos ingresados aceptados

La tasa de éxito corresponde al porcentaje de casos resueltos con acuerdo en relación con el total de casos ingresados a tramitación. Los casos con acuerdo son aquellos declarados como tal por el mediador e informado a las instituciones de esa forma. Los casos ingresados a tramitación, son aquellos en los que se cumplieron las formalidades descritas en el reglamento respectivo por parte de los requirentes.

Para el análisis de las diferencias en las tasas de éxito entre las mediaciones realizadas ante el consejo de defensa del estado y las intermediadas por la superintendencia de salud, se utilizó la prueba no paramétrica de Mann Withney con $p<0,05$.

\section{RESULTADOS}

Durante el período 2005-2009 se realizaron 4709 procedimientos de mediación en salud, de los cuales 3411 se realizaron ante el consejo de defensa del estado, por conflictos con prestadores públicos, y 1298 se realizaron por intermedio de la Superintendencia de salud, por situaciones derivadas de las prestaciones de salud privadas.

La tasa de éxito medio de los procedimientos de mediación del período 2005-2009 fue de 15,92\%, con valores superiores en los procedimientos realizados ante el consejo de defensa del estado, con una tasa media de éxito de $17,94 \%$, mientras que en los procedimientos intermediados por la Superintendencia de Salud fue de un 10,63\% para el mismo período, estas diferencias resultaron estadísticamente significativas $(p<0,05)$. El detalle de la tasa de éxito por año en ambos sistemas se encuentra en la Tabla I.

\section{DISCUSIÓN}

El establecimiento de un mecanismo prejudicial y no adversarial de resolución de conflictos en salud a partir del año 2005 y dentro del marco de la Ley 19.966 
Tasa de éxito de la mediación en salud en Chile entre los años 2005 y 2009 en el Sistema de Salud Público y Privado.

\begin{tabular}{ccccccc}
\hline & \multicolumn{2}{c}{$\begin{array}{c}\text { Número de casos } \\
\text { ingresados aceptados }\end{array}$} & \multicolumn{2}{c}{$\begin{array}{c}\text { Número de casos con } \\
\text { acuerdo a través de } \\
\text { mediación }\end{array}$} & \multicolumn{2}{c}{$\begin{array}{c}\text { Tasa de éxito del } \\
\text { procedimiento de } \\
\text { mediación }\end{array}$} \\
\cline { 2 - 7 } Año & $\begin{array}{c}\text { Sist. } \\
\text { Salud } \\
\text { Pública }\end{array}$ & $\begin{array}{c}\text { Sist. } \\
\text { Salud } \\
\text { Privada }\end{array}$ & $\begin{array}{c}\text { Sist. Salud } \\
\text { Pública }\end{array}$ & $\begin{array}{c}\text { Sist. Salud } \\
\text { Privada }\end{array}$ & $\begin{array}{c}\text { Sist. Salud } \\
\text { Pública }\end{array}$ & $\begin{array}{c}\text { Sist. Salud } \\
\text { Privada }\end{array}$ \\
\hline 2005 & 297 & 67 & 38 & 4 & 12.79 & 5.9 \\
2006 & 538 & 205 & 90 & 25 & 16.7 & 12.195 \\
2007 & 597 & 247 & 113 & 28 & 18.92 & 11.33 \\
2008 & 959 & 359 & 151 & 43 & 15.74 & 11.97 \\
2009 & 1020 & 420 & 220 & 38 & 21.56 & 9.04 \\
$\begin{array}{c}\text { Total periodo } \\
\text { 2005-2009 }\end{array}$ & $\mathbf{3 4 1 1}$ & $\mathbf{1 2 9 8}$ & $\mathbf{6 1 2}$ & $\mathbf{1 3 8}$ & $\mathbf{1 7 . 9 4}$ & $\mathbf{1 0 . 6 3}$ \\
\hline
\end{tabular}

ha posibilitado la búsqueda de soluciones entre las partes involucradas con la facilitación de un mediador. En nuestro estudio describimos una baja tasa de éxito general de estos procedimientos, por lo que a pesar del mecanismo de mediación vigente, una gran mayoría de los reclamos relacionada con la percepción del daño o vulneración de derechos en salud no es resuelta de esta forma y termina aumentando la insatisfacción del usuario o judicializando el conflicto.

El análisis de las causas de la baja tasa de éxito parece ser multifactorial, primero, el hecho que el derecho a reclamo pueda ejercerse desde la precepción usuaria que sus derechos han sido vulnerados. Esto genera que los prestadores sean llevados a procedimientos de mediación, que en el caso de los prestadores públicos están obligados a aceptar, sin un filtro mínimo de veracidad del hecho. En segundo lugar, la obligatoriedad del procedimiento, como paso previo a cualquier acción judicial, es contradictorio con el carácter voluntario de los acuerdos (Gómez, 1999; Vargas, 2008). Al respecto se estipula que ningún tribunal podrá tramitar una acción civil por daños contra prestadores institucionales públicos o sus funcionarios, si el demandante no ha recurrido previamente a esta mediación, dado que se establece que el sistema es obligatorio e ineludible (Ley 19.966; Ley 20.015; MINSAL). Un tercer aspecto parece estar en el marco jurídico que crea el procedimiento de mediación para conflictos en salud, ya que este se enmarca dentro de la ley de garantías en salud (Ley 19.966), conocida también como ley AUGE, posteriormente llamada ley GES. En este contexto, induce confusión que el procedimiento de mediación no se refiera exclusivamente a reclamos por dichas garantías, sino que para todas las relaciones entre prestadores y usuarios de los sistemas de salud. Un cuarto aspecto tiene que ver con las características del mediador, de acuerdo al reglamento de mediación, los requisitos para ser mediadores incluyen poseer título profesional de una carrera de a lo menos 10 semestres de duración, 5 años de experiencia laboral y no haber sido condenado, por lo que personas de ámbitos diferentes a los de la salud pueden constituirse en mediadores, lo que podría dificultar el diálogo entre las partes.

Al analizar los procedimientos de mediación originados por reclamos contra prestadores públicos y compararlos con lo que ocurre en relación a los prestadores privados observamos que el número de mediaciones fue significativamente mayor en el ámbito público, esto se relaciona, probablemente, con el mayor número de prestaciones que realiza el sector público y también con el carácter gratuito de los procedimientos de mediación ante el consejo de defensa del estado.

Las tasas de éxito de los procedimientos de mediación fueron bajos en el sistema público y privado, aunque significativamente mayores en el sistema público, lo que sugiere una mayor voluntad por encontrar salidas alternativas a los conflictos en el sector público, sumado a una pobre distribución regional de los mediadores registrados ante la superintendencia de salud.

Finalmente consideramos que el mecanismo de mediación prejudicial puede constituirse en una alternativa real para la resolución no adversarial de los conflictos en salud y que sus tasas de éxito pueden mejorar en la medida que se mejore la información y compromiso de los actores involucrados en la búsqueda de soluciones alternativas a los problemas derivados de la atención en salud. 
CANDIA, P. \& SUAZO, G. I. Success rate of pre-trial mediation system for health damage in Chile between 2005 and 2009. Int. J. Odontostomat., 5(3):300-303, 2011.

ABSTRACT: Pre-trial mediation in health damage was established in 2004 by 19.966 law which established the system of explicit guarantees of health in Chile. This procedure was established as a non-adversarial mechanism for conflict resolution in health. We searched the databases of the mediation procedures of the state defense council and the superintendent of health made between 2005 and 2009, we calculated the success rate, considered as the percentage of cases with agreement on the total number of cases accepted for mediation. The average success rate was $15.92 \%$, with higher values in the procedures performed at the State Defense Council, with $17.94 \%$, while in the intermediate by the Superintendency of Health was a $10.63 \%$ for the same period, these differences were statistically significant $(p<0.05)$. We discuss the likely causes of the low success rate and possible differences in the mediation for damages in the public and private health.

KEY WORDS: mediation, law 19.966, AUGE law, conflicts in health, deontology.

\section{REFERENCIAS BIBLIOGRÁFICAS}

Barría, G. P. Mediación por daños en salud del CDE: aporte a la cultura de la Paz. Revista de Derecho (CDE), 14:503, 2005.

Barría, P. \& Saint George, C. Modelo Mexicano de arbitraje médico: un aporte a la calidad de la medicina y a la comunicación entre médicos y pacientes. Revista de Derecho (CDE), 17:151-78, 2007.

CDE (Consejo de Defensa del Estado). 2011. Disponible en: http://www.cde.cl/portal.

Gómez, P. Propuestas y reflexiones éticas acerca del ejercicio de la mediación en Chile como forma de resolución no adversarial de los conflictos. Revista de Derecho de la Pontificia Universidad Católica de Valparaíso, 20:34375, 1999.

Gutiérrez, A. M. J. La Mediación en Salud y el acceso a la Justicia. Revista de Derecho (CDE), 20:111-37, 2008.

Ley n 19.966. Régimen General de Garantías en Salud. Diario Oficial, 3 de Septiembre, 2004. Disponible en: http:/ /www.bcn.cl/leyes/pdf. actualizado/229834.pdf.

Ley $n^{\circ} 20.015$. Modifica la Ley 18.433 de la Ley de Instituciones de Salud Previsional (ISAPRE). Diario Oficial, 20 de mayo, 2005. Disponible en: http://www.bcn.cl/leyes/ pdf/actualizado/238102.pdf.

Méndez, C. A. Los recursos humanos de salud en Chile: el desafío pendiente de la reforma. Rev. Panam. Salud Pública, 26:276-80, 2009.

MINSAL. Decreto N 47. Reglamento de mediación por reclamos contra prestadores institucionales públicos de salud o sus funcionarios y prestadores privados de salud. Diario Oficial, 23 de Junio de 2005. Santiago, Ministerio de Salud, 2005
Rodríguez, P. P. La experiencia francesa en mediación. Revista de Derecho (CDE), 19:43-92, 2008.

Superintendencia de Salud. 2011. Disponible en: http:// www.supersalud.gob.cl/568/w3-propertyvalue-3340.html.

Urriola, R. Chile: protección social de la salud. Rev. Panam. Salud Publica, 20:273-86, 2006.

Vargas, P. M. Mediación Obligatoria. Revista de Derecho (Valdivia), 21:183-202, 2008.

Dirección de correspondencia:

Prof. Dr. Iván Suazo Galdames

Departamento de Ciencias Básicas Biomédicas

Facultad de Ciencias de la Salud

Universidad de Talca, Talca

CHILE

Email: isuazo@utalca.cl

Recibido : 26-09-2011

Aceptado: 27-10-2011 